\title{
Effets de la charge des édifices volcaniques sur la propagation de structures régionales compressives : exemples naturels et modèles expérimentaux.
}

\section{Effects of volcanic loading on regional compressive structures: new insights from natural examples and analogue modelling.}

\author{
Yannick Branquet* and Benjamin Van Wyk de Vries \\ Département «Géosciences », université Blaise-Pascal, 5, rue Kessler, 63038 Clermont- \\ Ferrand, France \\ * Present Address : UMR6113 - ISTO
}

\section{Résumé}

Nous présentons ici des exemples naturels d'édifices volcaniques coniques reposant sur un substratum fragile, soumis à une compression régionale ainsi que des résultats expérimentaux. Nous montrons que la charge de l'édifice induit une perturbation de la déformation régionale se traduisant par une déflexion et une horizontalisation des structures compressives régionales. Le contrôle tectonique est de nature topographique. Nous discutons ensuite certaines conséquences, en particulier concernant l'étalement gravitaire des volcans.

\section{Abstract}

We present natural examples and experimental models of volcanic cones located above brittle substratum undergoing regional compressive deformation. The volcanic loading induces a strain partitioning involving deflection and flattening of regional compressive structures. The main control is the topographic load. Anticlinal thrust ridges, observed around many volcanoes, have generally been interpreted as being due to gravitational spreading; however, this study shows that this is not necessarily the case, as they can also be a symptom of regional compression.

Mots-clé: Atacama; Neuquén; regional compression; thrust and fold deflection; 'décollement'; sandbox experiments

Mots-clé: Atacama; Neuquén; compression régionale; déflexion; décollement; chevauchements; plis; modèles analogiques 


\section{Abridged version}

\section{Introduction}

The effects of volcanic loading on regional fault patterns has been studied for rifts [18] and strike-slip zones [19]: for rifts, normal faults are usually captured and bent into the cone whereas in the case of strike-slip zones, normal faults are generated within the cone, inducing pull-apart extension. Numerous volcanic regions are also subjected to regional contraction ( figure 1). For example, in the southern Atacama of northern Chile (figure 1A), thrusts are present and related to contractional neotectonics [7]. A small volcanic cone is trapped in the thrust system; Quaternary ignimbrites (underlying the cone) are deformed by west-verging thrusts trending broadly north-south. The thrust traces display a clear westward arcuate deflection at the western cone foot. Southward, one of the thrusts curves into a NW-SE trending lateral ramp, which corresponds to a left lateral strike-slip thrust. Thus, the thrusts curve around the cone. A similar pattern of folds and thrusts is found in the Neuquén basin (Argentina). Here the thrusts are deflected around the Tromen volcano (figure 1B), which is of Tertiary to Holocene age [4 and 5]. Folds and thrusts of the region ( figure 1B) have been formed or reactivated during Neogene compression [9]. This deflection had been formerly interpreted as the result of the Pleistocene-Holocene collapse of the Tromen edifice [4]. In accordance with Ornelas Marques and Cobbold [13], we show that it results from the interaction between volcanic loading and regional compression.

\section{Experiments}

A set of experiments has been performed using simple analogue sandbox apparatus (figure $2 \mathrm{~A}$ and table). Our models are purely brittle: (1) the substratum is composed of dry sand, currently used in analogue modelling [6 and 21] and that fails according to the MohrCoulomb criterion of brittle failure - the density of the dry sand used is $1.4 \cdot 10^{3} \mathrm{~kg} \cdot \mathrm{m}^{-3}$, its angle of internal friction is $30^{\circ}$ and its cohesion is $0( \pm 15) \mathrm{Pa}$ at zero normal stress [16]; (2) the cone is represented by an alternation of sand layers and mixed layers $(20 \% \mathrm{wt}$ plaster + $80 \%$ wt sand, density: $1.3 \cdot 10^{3} \mathrm{~kg} \cdot \mathrm{m}^{-3}$, angle of internal friction: $33^{\circ}$ and cohesion about $40-60$ $\mathrm{Pa}$ at zero normal stress [15]) in order to generate contrasts of mechanical strength in the cone as within a natural stratovolcano. According to Van Wyk de Vries and Merle [18], the model is 100000 times weaker than natural prototypes and length is 60000 times smaller. Figure $2 B$ presents a model without a cone. The shortening is accommodated by a pop-up, bounded by a permanent major thrust (CMP) and by a system of transient thrusts (CT) (time sequence $1,2,3, \ldots, 7$ in figure $2 B$ ). 

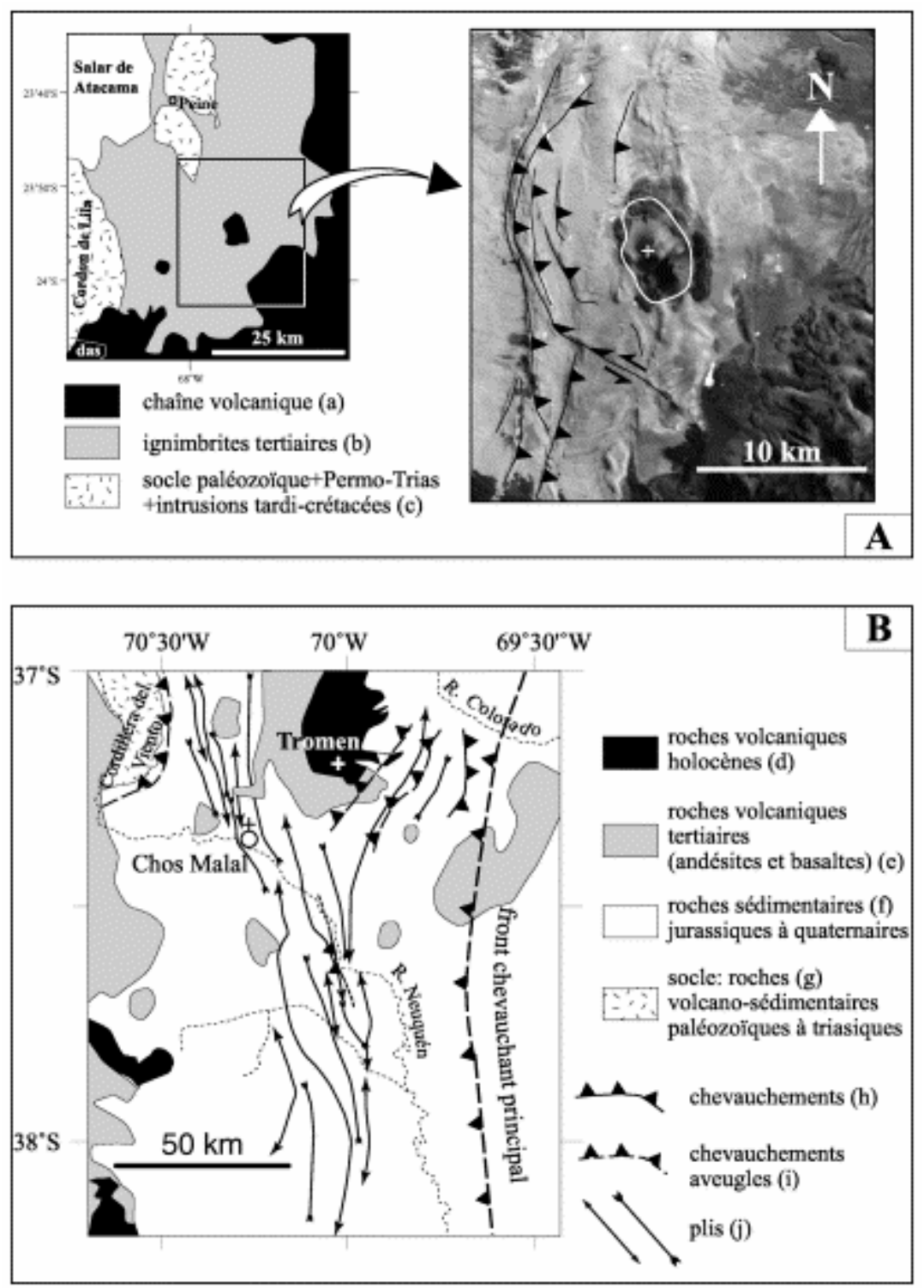

Figure 1. Édifices volcaniques soumis à une tectonique compressive. A. Sud-Est du Salar de Atacama au Chili, région de Peine. Interprété d'après l'analyse de photos Landsat TM et d'études de terrain. das : Lobe septentrional du débris d'avalanche du volcan Socompa [20]. B. Carte structurale simplifiée de la région de Chos Malal, dans le bassin de Neuquén, en Argentine. Interprétée d'après l'analyse de photos Landsat TM et de [4].

Volcanic constructs undergoing regional compressive tectonics. A. Southeastern edge of the Salar de Atacama, Peine area, Chile. Interpreted from Landsat TM image and field work. das: northern part of the Socompa debris avalanche [20]. a: Volcanic chain; b: Tertiary ignimbrites; c: Palaeozoic basement + Permo-Triassic rocks + Late Cretaceous intrusions. B. Simplified structural map of the Chos Malal area in the Neuquén basin, Argentina. Interpreted from Landsat TM image and [4]. d: Holocene volcanic rocks; e: Tertiary volcanic rocks; f: Jurassic to Quaternary sedimentary rocks; $\mathbf{g}$ : basement (Palaeozoic to Triassic volcano-sedimentary rocks); $\mathbf{h}$ : thrusts; i: blind thrusts; $\mathbf{j}$ : folds. 


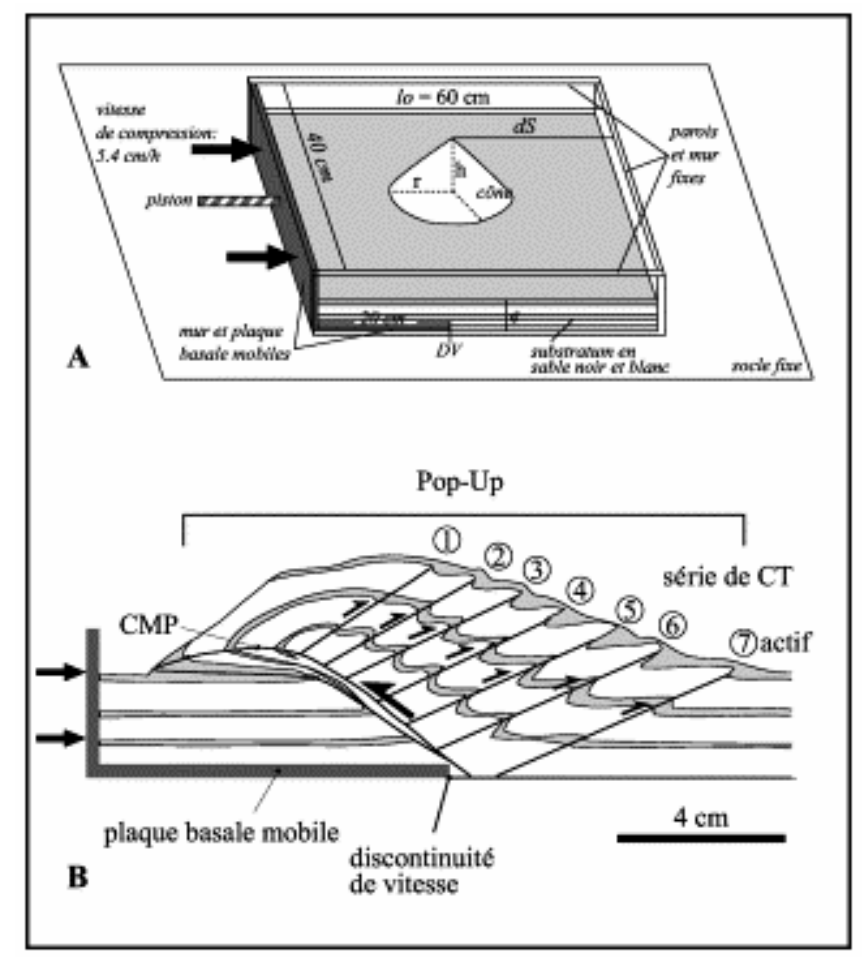

Figure 2. A. Dispositif expérimental. Le modèle est constitué d'un ensemble socle-mur-paroi fixe, sur lequel se déplace une plaque basale mobile solidaire d'un mur mobile. L'ensemble plaque basale et mur mobile est poussé par un piston actionné par un moteur pas-à-pas, commandé par un ordinateur. Ce dispositif, soumis à un raccourcissement perpendiculaire appliqué au mur mobile, génère une discontinuité de vitesse au front de la plaque mobile, qui localisera la déformation [12 and 17]. $e$ : Épaisseur du substratum; $h:$ hauteur du cône ; $r$ : rayon du cône ; $d S$ : distance mur fixe-centre du cône ; $D V$ : discontinuité de vitesse $\mathbf{B}$. Modèle témoin, sans cône sus-jacent. Cette structure en pop-up, décrite et reproduite dans de nombreux travaux expérimentaux (par exemple, [17]), est limitée, du côté piston, par un chevauchement majeur permanent (CMP) et, du côté mur fixe, par un système de chevauchements transitoires (CT). CMP et CT se forment au niveau de la discontinuité de vitesse. Lors de la progression de la discontinuité de vitesse vers le mur fixe, les CT se créent et s'inactivent successivement, étant alors transportés passivement le long du CMP (chronologie 1,2,3,..,7). Pour un même taux de raccourcissement et suivant des épaisseurs de substratum croissantes : la largeur du pop-up augmente, le nombre de CT diminue, alors que leur espacement augmente, tandis que le pendage du CMP en profondeur demeure constant $\left(30\right.$ à $\left.35^{\circ}\right)$. Le pendage d'initiation d'un CT est de l'ordre de 25 à $30^{\circ}$.

The experimental apparatus is composed of a basal mobile plate displaced perpendicular to a fixed part (basement, side and frontal walls). The basal plate is fixed to a mobile wall pushed by a computer-controlled screw-jack. The front of the mobile basal plate represents a velocity discontinuity $(D V)$ that localizes the deformation [12 and 17]. $e$ : substratum thickness; $h$ : cone height; $r$ : cone radius; $d S$ : distance fixed frontal wallcone centre. B. Model without cone. For a same shortening rate and for increasing substratum thickness: the popup thickness increases, the CT number decreases and the CT spacing increases. The CMP dip remains constant at depth $\left(30\right.$ to $\left.35^{\circ}\right)$ and the $\mathrm{CT}$ incipient dip is about 25 to $30^{\circ}$. 
Experimental parameters presented in figure 3. See figure 2 for abbreviations.

\begin{tabular}{lllclll}
\hline Expérience & $\begin{array}{l}e \\
(\mathrm{~cm})\end{array}$ & $\begin{array}{l}h \\
(\mathrm{~cm})\end{array}$ & $\begin{array}{l}r \\
(\mathrm{~cm})\end{array}$ & $\begin{array}{l}d S \\
(\mathrm{~cm})\end{array}$ & $\begin{array}{l}l o \\
(\mathrm{~cm})\end{array}$ & $\begin{array}{l}\text { vitesse } \\
\left(\mathrm{cm} \cdot \mathrm{h}^{-1}\right)\end{array}$ \\
\hline VEC9 & 5 & 5,5 & 8 & 27 & 60 & 5,4 \\
VEC10 & 2,7 & 5,5 & 8 & 41 & 60 & 5,4 \\
VEC11 & 1,8 & 6,8 & 10 & 38 & 60 & 5,4
\end{tabular}

\section{Results}

The three experiments show that the CT and CMP undergo a clear arcuate deflection associated with the development of transpressive areas (figure 3). These zones of transpression form later than pure thrusting segments and correspond to a fault linkage area (e.g., VEC11). For important $h / e$ (height of volcano/thickness of sand layer) ratios, the structural deflection is associated with a flattening of the thrusts, inducing the formation of true brittle 'décollements' (VEC11, cross-section $\mathrm{BB}^{\prime}$ ). The linkage between the thrust segments occurs through the formation of a duplex (figure 3). In the experiment VEC9, the cone lies above the monovergent $\mathrm{CT}$ system. The first $\mathrm{CT}$ does not propagate below the cone and is relayed by the formation of a conjugate back-thrust. Thus, the dip and orientation of the cone slope controls the vergence of regional thrusts. So, as suggested by Ornelas Marques and Cobbold [13], the main deformation control is the topography, in this case the volcanic cone itself.

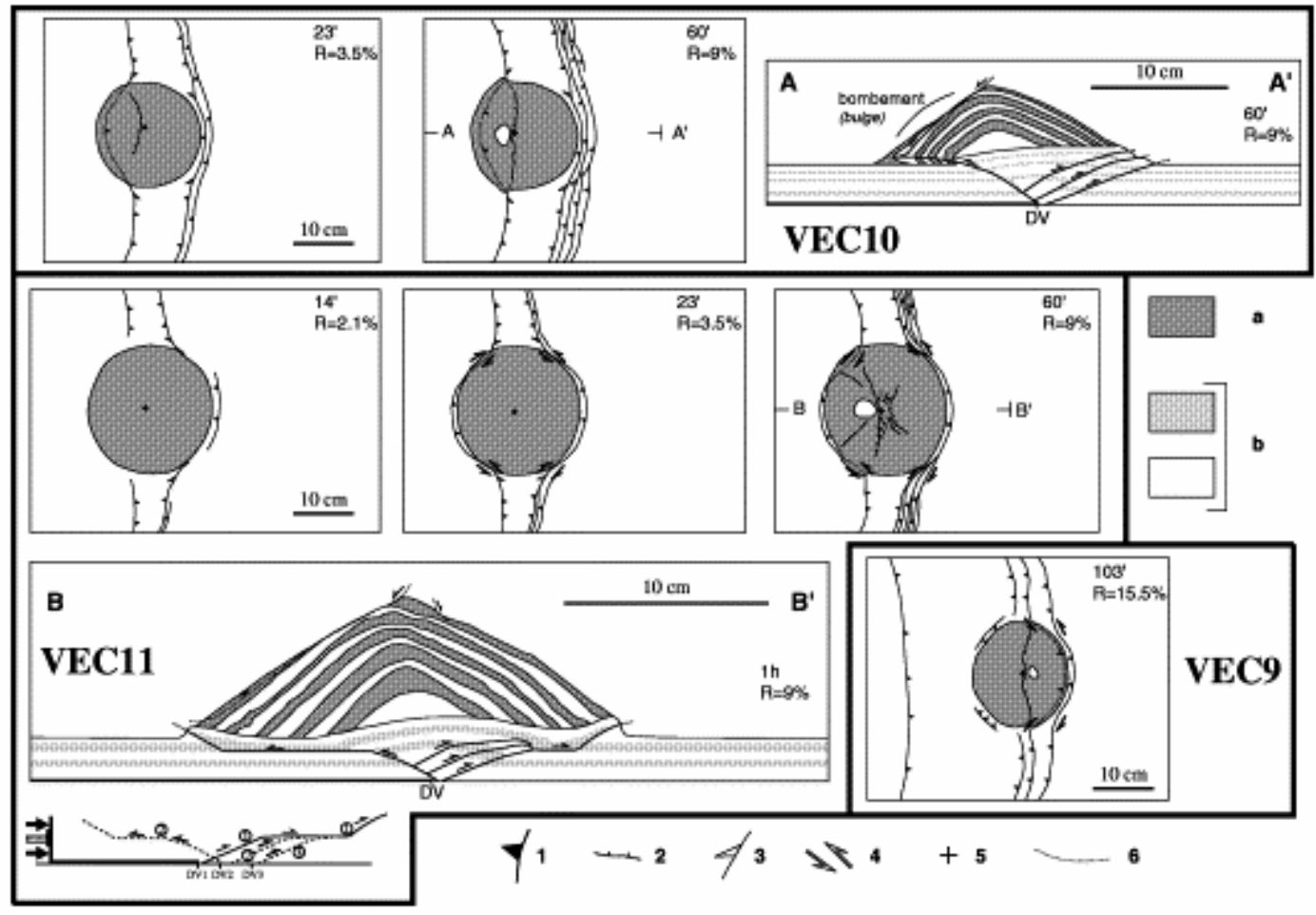

Figure 3. Résultats des expériences VEC10, VEC11 et VEC9. Explications dans le texte. $R$ : raccourcissement ; $D V:$ discontinuité de vitesse ; $\mathbf{a}:$ mélange sable + plâtre $; \mathbf{b}:$ sable $; \mathbf{1}:$ chevauchement ; $\mathbf{2}:$ faille normale ; $\mathbf{3}$ : 
décro-chevauchement ; 4 : zone de relais décro-chevauchante ; 5 : point culminant du cône ; 6 : structure aveugle.

Results from experiments VEC10, VEC11 and VEC9. See text for explanation. $R$ : shortening; $D V$ : velocity discontinuity; a: sand + plaster mixture; b: sand; 1: thrust; 2: normal fault; 3: strike-slip thrust; 4: transpressive zone; 5 : top of the cone; $\mathbf{6}$ : blind structure.

In contrast with rift, strike-slip and steep basement fault situations [11, 18 and 19], the cones undergoing regional compression are not affected by faults with dips steeper than the edifice slope. Moreover, in VEC10, the CMP triggers the formation of a bulge on the cone that becomes unstable. The gravity equilibrium is then reached by flank collapse along a superficial normal fault. Unstable bulging and associated flank collapse have also been described in the experiments of basement fault reactivation below volcanoes [11]. However, in our experiments, we identified a normal fault that is clearly connected to the CMP. This appears to be a very similar process to the syn-thrusting collapse of ramp anticlines in deformed foreland basins [1]. We think that this process could be responsible for large sector collapses. Indeed, in the same thrust belt as our Chilean example, the major Socompa debris avalanche may have been caused by this process [20].

\section{Discussion}

The experiment VEC10 (figure 3) shows that an arcuate anticline ridge can form at an edifice without being related to any volcanic spreading process (e.g., the fault-propagation fold of Misterbianco-Catania, south of Etna [2, 3 and 10]). Thus, for such basal anticline ridges, their arcuate morphology and their lack of connection with regional structures cannot be exclusively assigned to volcano spreading: regional compression is an alternative cause that must be taken into account. This study has direct implications on the geometry of accretionary prisms with mud volcanism. As some mud volcanoes are up to $10 \mathrm{~km}$ wide [14], their load should be able to deflect faults during thrust propagation [8].

\section{Conclusions}

The natural examples can be interpreted from our experimental results.

Nearby a conical construct (volcano, mud volcano...), the contractional structures propagate by deflection around the cone.

The deflection is associated with the development of transpression zones linking the firstformed thrusts. In these zones, regional thrusts coalesce, forming classic duplexes.

Below the cone, the regional thrusts are flattened to form horizontal 'décollements' that decouple sand layers below the edifice.

This strain partitioning (deflection and decoupling at depth) is mainly controlled by the volcanic topography.

In contrast with rifts and strike-slip regional tectonics, contractional tectonics may not generate steeply dipping structures within the cone. However, flank collapses can evolve with superficial normal faults branching from regional thrusts. 
Deflection and flattening appear to be a powerful tool for the dating of regional deformation relative to the age of volcanic edifices.

Regional compression has to be also considered when interpreting anticline ridges at the base of a volcano: these ridges are not exclusively symptomatic of volcanic spreading.

\section{Introduction}

L'effet de la charge des édifices volcaniques sur la tectonique régionale a été abordé dans le cas des rifts [18] et des zones de décrochement régionales [19] : dans les deux cas, le poids des édifices réoriente localement le champ de contrainte régional en induisant de l'extension et en la focalisant dans le cône déformé par des failles normales. Les failles normales régionales sont captées par le cône dans le cas d'un rift, alors que, dans le cas des décrochements, elles sont générées au sein même du cône, induisant une extension de type pull apart. Cette extension, localisée dans les cônes, opère alors comme un rétrocontrôle tectonique positif sur l'ascension des magmas lors de l'ouverture de sites dilatants.

Cependant, de nombreux édifices volcaniques sont soumis à des champs de contraintes régionaux compressifs (figure 1). La figure $1 \mathrm{~A}$ montre un cône volcanique de dimension réduite, reposant sur des ignimbrites quaternaires. Vers l'ouest, les ignimbrites sont déformées par un système de chevauchements à vergence ouest, d'orientation générale nord-sud, et compatibles avec la néotectonique compressive reconnue dans cette zone [7]. La trace de ces chevauchements affiche une nette déflexion arquée et convexe vers l'ouest, au front de l'édifice volcanique. L'un de ces chevauchements s'arque et se prolonge vers le sud en rampe latérale décro-chevauchante senestre, d'orientation NW-SE. La trace des chevauchements et des plis régionaux autour du volcan Tromen, dans le bassin de Neuquén en Argentine (figure 1B), décrit également une remarquable déflexion, divergente de part et d'autre de l'édifice. Le massif volcanique du Tromen est d'âge Tertiaire à Holocène [4 and 5]. Bien qu'une partie des structures se soient formées lors d'une déformation transpressive dextre dès l'Éocène [5], une tectonique compressive néogène s'est superposée, réactivant et formant ainsi les chevauchements et les plis observables sur la figure $1 B$ [9].

Cette déflexion des structures compressives autour des volcans s'observe assez fréquemment, décrivant ainsi une morphologie "en oil », dans laquelle les chevauchements et les plis semblent se propager en "évitant » le cône. La déflexion des structures régionales de part et d'autre du Tromen avait préalablement été interprétée comme résultant de l'effondrement pléisto-holocène du volcan [4]. Comme d'autres auteurs l'ont suggéré [13], nous montrons ici que cette déflexion résulte de l'interaction entre la charge du volcan et un champ de contrainte régional compressif.

\section{Dispositif et procédure expérimentaux}

Nous avons réalisé une série d'expériences simples modélisant une compression affectant un substratum purement fragile, surmonté d'un édifice conique également fragile (figure $2 A$ ). Le substratum est composé de sable sec, analogue classiquement utilisé des roches à comportement fragile [6 and 21], et dont la rupture fragile suit le critère de Mohr-Coulomb. La masse volumique du sable sec utilisé est de $1,4 \cdot 10^{3} \mathrm{~kg} \cdot \mathrm{m}^{-3}$, son angle de frottement interne de $30^{\circ}$ et sa cohésion de $0( \pm 15)$ Pa pour des contraintes normales quasi nulles (i.e. à proximité d'une surface libre) [16]. Un modèle témoin sans cône est présenté sur la figure $2 B$. 
Afin de modéliser les contrastes mécaniques d'un stratovolcan et de prendre en compte les écarts de cohésion observés sur les volcans naturels (de $10^{5}$ à $10^{6} \mathrm{~Pa}$ [11]), les cônes utilisés sont hétérogènes et composés d'une alternance de couches de sable sec (cf. caractéristiques cidessus) et de couches d'un mélange sable + plâtre (20\% en poids). Un tel mélange a également un comportement de type Mohr-Coulomb et possède une masse volumique de $1,3 \cdot 10^{3} \mathrm{~kg} \cdot \mathrm{m}^{-3}$, un angle de frottement interne de $33^{\circ}$ et une cohésion de 40-60 Pa sous une contrainte normale proche de zéro [15].

Les rapports d'échelle entre modèle et système géologique naturel sont du même ordre que ceux de Van Wyk de Vries et Merle [18], à savoir : $1,6 \cdot 10^{-5}$ pour les longueurs $(1 \mathrm{~cm}$ représente $600 \mathrm{~m}$ ), $10^{-5}$ pour les forces et les contraintes (le modèle est 100000 fois moins résistant que le système naturel). Les modèles étant purement fragiles, la déformation est indépendante du temps.

Les paramètres initiaux des trois expériences de la figure 3 sont présentés dans le tableau. La vitesse de compression est constante $\left(5,4 \mathrm{~cm} \cdot \mathrm{h}^{-1}\right)$ et identique pour les trois cas. Avant d'exercer la compression, les cônes ont été construits et localisés précisément, afin d'être affectés, soit par le système chevauchements transitoires (CT) + chevauchement majeur permanent (CMP), soit par le système de CT seulement. Après mouillage du modèle, des coupes sériées sont réalisées.

\section{Résultats expérimentaux}

\subsection{Structures régionales}

Lors des trois expériences, les chevauchements régionaux (CT ou CMP) subissent clairement une déflexion de part et d'autre du cône et acquièrent une morphologie arquée, concentrique par rapport au cône (figure 3). Cette déflexion s'accompagne du développement de zones de relais décro-chevauchantes, reliant les segments purement chevauchants. Chronologiquement, ces zones transpressives se mettent en place après l'apparition des segments chevauchants. Ainsi, pour un faible raccourcissement $(2,1 \%)$, lorsque le rapport $h / e$ est important (VEC11) : (i) des segments chevauchants se développent à la base du volcan, sans connexion apparente avec les structures régionales ; (ii) la charge de l'édifice peut inhiber la propagation du CMP sous le cône. Ces zones transpressives présentent également une coalescence des accidents, allant jusqu'à ne former qu'un seul chevauchement, actif en permanence à la base du cône (cf. VEC11).

La déflexion des structures s'accompagne d'une horizontalisation des chevauchements sous le cône, allant jusqu'à produire, pour des rapports $h / e$ importants, de véritables décollements plats fragiles (coupe BB', VEC11). Le chevauchement à droite du cône étant resté unique et actif depuis la $14^{\mathrm{e}}$ minute (VEC11), nous interprétons la coalescence observée comme étant issue du développement d'un duplex (figure 3) de CT.

L'expérience VEC9 utilise uniquement le système de CT qui est mono-vergent, sans cône. Le premier CT formé (à vergence normale vers le mur fixe) ne se propageant pas sous le cône, c'est le rétro-chevauchement conjugué (à vergence vers le piston et formant deux segments à gauche de l'édifice représenté sur la figure 3) qui a accommodé la déformation sous le cône. La trace de ce premier CT passe à gauche du centre du cône, alors que celles du second et du troisième CT (sans rétro-chevauchement) passent à droite. Ainsi, la vergence des chevauchements affleurant au voisinage du cône apparaît contrôlée par l'orientation de la 
pente (vers la gauche ou vers la droite) du cône, à l'endroit où la trace du chevauchement régional intersecte l'édifice.

L'émergence des chevauchements juste à la base du cône, leurs vergences (cf. ci-dessus) et le parallélisme entre la trace arquée des chevauchements et les contours du cône, montrent que le principal contrôle de la déformation est topographique (c'est-à-dire ici, le relief). Ceci est en accord avec les travaux récents de Ornelas Marques et Cobbold [13]. Pour cette raison, l'analyse mécanique tridimensionnelle de la déformation est délicate. Le cône induit une zone sous-jacente, au sein de laquelle les contraintes principales sont réorientées. En deux dimensions sur la coupe BB', le $\sigma_{1}$ opérant lors de la formation des rampes ne semble pas influencé par le tenseur de contrainte du cône et correspond donc au $\sigma_{1}$ régional horizontal. Par contre, lors de la formation des décollements, le $\sigma_{1}$ opérant est composite (tenseur régional combiné au tenseur du cône) et subit une rotation d'environ $30^{\circ}$ suivant un axe horizontal.

\subsection{Déformation du cône}

En comparaison des rifts [18], des zones de décrochement régionales [19] et des failles de socle réactivées sous les volcans [11], les cônes soumis à une compression régionale ne semblent pas être affectés par des failles à pendage supérieur aux pentes de l'édifice. Le biseau limité par le CMP et la pente sommitale gauche du cône (par exemple, VEC10 et VEC11) est largement supercritique et subit donc une simple translation. Néanmoins, lorsqu'il n'affecte qu'une partie de l'interface cône-substratum, le soulèvement induit une augmentation de la pente du cône (cf. la pente gauche de VEC10), formant ainsi un large bombement. Ce bombement, gravitairement instable, occasionne le développement d'une faille normale superficielle, responsable de l'effondrement (et du rééquilibrage gravitaire) du flanc du cône. Ce processus, associant formation d'un bulge et effondrement de flanc a été étudié lors de la réactivation de failles de socle sous les volcans [11]. Cependant, lors de l'expérience VEC10, la faille normale est clairement connectée au CMP. En considérant un biseau supercritique instable gravitairement, ce mécanisme est strictement similaire à l'effondrement gravitaire syn-chevauchement des anticlinaux de rampe [1] : des failles normales à pendage vers l'avantpays viennent se brancher sur le décollement et affectent le flanc instable de l'anticlinal. Nous pensons que ce mécanisme, impliquant des failles normales dans un volcan associées à des chevauchements régionaux, intervient lors de la genèse d'effondrements sectoriels (cf. l'effondrement sectoriel et l'avalanche de débris du volcan Socompa [20]) et mérite de plus larges investigations.

\section{Discussion}

Au début de l'expérience VEC11 (figure 3), un chevauchement légèrement arqué s'est formé devant le cône, sans qu'il soit apparemment connecté aux CT en surface. Ce type de structure, formant des rides anticlinales concentriques, se rencontre à la base de nombreux édifices volcaniques (comme, par exemple l'anticlinal de propagation de faille de MisterbiancoCatania, sur le flanc sud de l'Etna [3]). Elles sont couramment interprétées comme résultant de l'étalement gravitaire du volcan (volcanic spreading) [2,3 and 10] : l'extension affectant le cône est accommodée par des rides compressives concentriques affectant le substratum à la base de l'édifice. Nos expériences montrent clairement qu'une origine tectonique régionale est également une alternative à prendre en compte dans l'interprétation de ces rides anticlinales basales. Plus précisément, l'existence de ces rides basales, leur morphologie arquée et leur 
apparente déconnexion par rapport aux structures régionales ne sont pas le fait exclusif de l'étalement gravitaire.

Cette étude a également des implications directes sur la géométrie des prismes d'accrétion associés à un volcanisme boueux. En effet, les volcans de boue, comme ceux de la zone Olympie en Méditerranée, présentent des dimensions importantes (jusqu'à $10 \mathrm{~km}$ ) [14]. Leur charge est donc susceptible de perturber la propagation des structures compressives. En particulier, nous suspectons que les structures circulaires décrites autour de ces édifices [8], correspondent à la déflexion «en œ il » des chevauchements et des plis, de part et d'autre du volcan de boue.

\section{Conclusions}

Les exemples naturels présentés peuvent être interprétés à la lumière de nos modèles expérimentaux. Les conclusions sont les suivantes.

La propagation de structures compressives régionales (chevauchements, plis par propagation de faille) à proximité d'un édifice conique (volcans, volcans de boue...) s'accompagne d'une déflexion de ces structures de part et d'autre du cône.

Cette déflexion se forme par la mise en place de zones de relais décro-chevauchantes, reliant les segments purement chevauchants apparus en premier.

Au niveau de ces zones transpressives, les chevauchements subissent une coalescence, induite par la formation de duplex.

Cette déflexion s'accompagne d'une horizontalisation des chevauchements sous l'édifice, allant jusqu'à la formation de décollements plats.

Cette répartition de la déformation (déflexion et découplage en profondeur) est contrôlée par la topographie, c'est-à-dire, ici, le relief.

Contrairement aux rifts et aux décrochements, la compression régionale ne semble pas générer de structures à fort pendage dans le cône. Cependant, des effondrements superficiels de flanc semblent impliquer des failles normales se branchant sur les chevauchements régionaux.

Déflexion et horizontalisation observées en carte et/ou en coupe constituent un puissant outil d'aide à la datation de la déformation régionale par rapport à l'âge des édifices volcaniques, les études néotectoniques apparaissant alors comme domaine d'application immédiat.

Les anticlinaux et les ceintures de chevauchements concentriques affectant le substratum à la base d'un édifice volcanique ne sont donc pas exclusivement symptomatiques de l'étalement gravitaire de l'édifice (volcanic spreading) : une compression régionale contemporaine du cône, qui génère des structures analogues (connectées ou non à de grandes structures régionales) doit être potentiellement considérée. 


\section{Bibliographie}

1. M. Bonini, D. Sokoutis, G. Mulugeta and E. Katrivanos , Modelling hanging wall accommodation above rigid thrust ramps. J. Struct. Geol. 22 (2000), pp. 1165-1179.

2. A. Borgia , Dynamic basis of volcanic spreading. J. Geophys. Res. 99 B9 (1994), pp. 17791-17804.

3. A. Borgia, L. Ferrari and G. Pasquarè , Importance of gravitational spreading in the tectonic and volcanic evolution of mount Etna. Nature 357 (1992), pp. 231-235.

4. V. Chauveau, B. Nivière, P.R. Cobbold, E.A. Rossello, J.-F. Ballard and H.T. Eichenseer, Structure of the Andean foothills, Chos Malal region, Neuquén Basin, Argentina. In: Third Int. Symp. Andean Geodyn., St. Malo, Ext. Abstr., , Éditions Orstom (1996), pp. 315-318.

5. P.R. Cobbold, M. Diraison and E.A. Rossello, Bituminen veins and Eocene transpression, Neuquén Nasin, Argentina. Tectonophysics 314 (1999), pp. 423-442.

6. E. Faugère and J.-P. Brun, Modélisation expérimentale de la distension continentale. $C$. $R$. Acad. Sci. Paris, série II 299 (1984), pp. 365-370.

7. S. Flint, P. Turner, E.J. Jolley and A.J. Hartley, Extensional tectonics in convergent margin basins: an example from the Salar de Atacama, Chilean Andes. Geol. Soc. Am. Bull. 105 (1993), pp. 603-617.

8. C. Huguen, Volcanisme boueux et déformation récente à actuelle au sein de la ride Méditérranéenne, d'après les données de la campagne Prismed II. Géologie Alpine 75 (1999), pp. 174-180.

9. E.E. Kozlowski, C.E. Cruz and C.A. Sylwan, Geologia estructural de la zona de Chos Mamla, Cuenca Neuquina, Argentina. In: (1996), pp. 15-26.

10. O. Merle and A. Borgia, Scaled experiments of volcanic spreading. J. Geophys. Res. 100 B6 (1996), pp. 13805-13817.

11. O. Merle, N. Vidal and B. Van Wyk de Vries, Experiments on vertical basement faults reactivation below volcanoes. J. Geophys. Res. 106 B2 (2001), pp. 2153-2162.

12. T. Nalpas, I. Györfy, F. Guillocheau, F. Lafont and P. Homewood, Influence de la charge sédimentaire sur le développement d'anticlinaux synsédimentaires. Modélisation analogique et exemple de terrain (bordure sud du bassin de Jaca). Bull. Soc. géol. France 1705 (1999), pp. 733-740.

13. F. Ornelas Marques and P.R. Cobbold, Topographic controls on deformation, partitioning, decoupling and vertical-axis rotations in thrust belts: insights from sandbox modelling, EUG XI Meeting, Strasbourg, France. J. Conf. Abs. (2001), p. 396.

14. A. Robertson, Mud volcanism on the Mediterranean Ridge: initial results of Ocean Drilling Program Leg 160. Geology 24 (1996), pp. 239-242. 
15. O. Roche, B. Van Wyk de Vries and T.H. Druitt, Sub-surface structures and collapse mechanisms of summit pit craters. J. Volc. Geotherm. Res. 105 (2001), pp. 1-18.

16. W.P. Schellart, Shear test results for cohesion and friction coefficients for different granular materials: scaling implications for their usage in analogue modelling. Tectonophysics 324 (2000), pp. 1-16.

17. J.J. Tondji Biyo, Chevauchements et bassins compressifs, influence de l'érosion et de la sédimentation, modélisation analogique et exemples naturels, thèse $3^{\mathrm{e}}$ cycle. Mém. Géosciences Rennes 59 (1995) 426 p .

18. B. Van Wyk de Vries and O. Merle, The effect of volcanic constructs on rift fault patterns. Geology 247 (1996), pp. 643-646.

19. B. Van Wyk de Vries and O. Merle, Extension induced by volcanic loading in regional strike-slip zones. Geology 2611 (1998), pp. 983-986.

20. B. Van Wyk de Vries, P.W. Francis, S. Self and L. Kesthelyi, Development of the Socompa Debris avalanche (N. Chile) from a spreading volcanic edifice. J. Volc. Geotherm. Res. 1053 (2001), pp. 225-248.

21. B. Vendeville, P.R. Cobbold, P. Davy, J.-P. Brun and P. Choukroune, Physical models of extensional tectonics at various scales. In: M.P. Coward, M.F. Dewey and P.L. Hancock, Editors, Continental Extensional TectonicsSpec. Pub. Geol. Soc. London (1987), pp. 95-107. 